\title{
Gibt es die postkritische Pädagogik auch moralinfrei?
}

\author{
Ein systemtheoretischer Nachvollzug \\ verschiedener Affirmationsformen auf den Ebenen \\ von Praxis, Semantik und Erziehungswissenschaft
}

Franz Kasper Krönig

Wenn Hodgson et al. (2017; 2022) in ihrem Manifest von postkritischer Pädagogik sprechen, scheinen sie nicht eine spezielle Pädagogik ins Spiel bringen $\mathrm{zu}$ wollen, sondern eine Bestimmung von Pädagogik überhaupt. Es geht sozusagen um das Pädagogische der Pädagogik. Postkritische Pädagogik ist mithin ein umfassender Definitionsversuch von Pädagogik, um nicht zu sagen: eine Wesensbestimmung oder Ontologie des Pädagogischen (vgl. Vlieghe/Zamojski 2020). Dass diese Bestimmung mit einer Abgrenzung arbeitet, verbindet sie mit jeder wissenschaftlichen Definition. Dass sich diese Abgrenzung auf die kritische Pädagogik bzw. Erziehungswissenschaft bezieht, kann als historischer Index dieser Definition gesehen werden. Während es sozusagen die historische Situation der Kritischen Pädagogik erforderte, sich von humanistischen Bildungstheorien abzuheben und dadurch das sichtbar zu machen, was eben diese verhüllten, hatten sich bspw. für die intersektionale oder postkoloniale Pädagogik Abrenzungsnotwendigkeiten gegenüber kritisch informierten Reformpädagogiken ergeben. Man muss keine historische Dialektik vertreten, um zu sehen, dass die jeweils historisch gewordenen Negationen nicht einfach ausgelöscht werden, sondern im nächsten Begriff enthalten bzw. auf- 
gehoben sind. Das Postkritische will also genau in diesem Sinne weder unkritisch noch antikritisch sein (vgl. Krönig 2020), sondern eine Stufe des Begriffs der Pädagogik erreichen, auf der die Abgrenzungen des Kritischen zum Humanistischen oder des Intersektionalen vom Kritischen enthalten sind, auf der aber eine neue Abhebung in den Fokus gerät, die das, was zuvor ausgeblendet war, aufblendet.

Will man diese Definition der Pädagogik nachvollziehen, stößt man allerdings auf das Problem, dass im Manifest mal von »Bildungstheorie« (Hodgson et al. 2022: 19), mal von »erziehungswissenschaftlichen Forschungen« (ebd.), meist von »die Pädagog*in« und immer wieder von einem »wir« gesprochen wird. Ist die postkritische Pädagogik also Bildungsphilosophie, Erziehungswissenschaft, pädagogische Profession oder pädagogische Praxis? Gut vorstellbar ist, dass die Autor*innen hierauf mit »ja« antworten würden - »all dies ist mit postkritischer Pädagogik gemeint«. Für eine begriffliche Analyse muss allerdings diese mögliche These der Einheit der Differenzen problematisiert und begründet, d.h. entproblematisiert werden. Leicht wäre es ansonsten, bei Formulierungen wie »Liebe für die Welt«, »Hoffnung«, »das Gute in der Welt « oder dem empathischen »wir« oder »unsere« rote Karten zu zeigen im Sinne einer Disqualifizierung der Disziplin aufgrund von Kitsch, Affirmation oder möglicherweise unhaltbarer Epistemologie. Kann die Bildungsphilosophie oder allgemeiner die Erziehungswissenschaft derartig normative und sogar emphatische Formulierungen als eigene zulassen? Natürlich kann die Erziehungswissenschaft alle möglichen Semantiken (man denke nur an religiöse Semantiken Fröbels oder moralische Semantiken Korczaks) zum Forschungsgegenstand machen - als begriffliches Inventar ihrer disziplinären Operationsweise sind diese allerdings ohne Frage nicht tauglich (vgl. Binder/Heidenwag 2013). Die Erziehungswissenschaft kann, einfach gesagt, Bezüge auf Gott und das Gute im Menschen nicht als wissenschaftliche Beiträge zulassen. Müsste dies nicht auch für Liebe, Hoffnung und das Gute gelten?

Im Folgenden soll daher ausdifferenziert werden, was die postkritische Pädagogik mit »Pädagogik«, »Bildung«, »Bildungstheorie« bzw. »erziehungswissenschaftliche Forschung« meinen könnte und 
was auf diesen Ebenen jeweils unter "postkritisch« zu verstehen wäre. Erst auf dieser Grundlage kann die These einer Einheit der postkritischen Pädagogik, die möglicherweise diese Differenzen enthält, diskutiert werden. Der Versuch besteht darin, in systemtheoretischer Manier verschiedene Beobachtungs- und Reflexionsverhältnisse $\mathrm{zu}$ unterscheiden. Es werden also jeweils Unterscheidungen innerhalb des Pädagogischen dargestellt. Die erste Unterscheidung, ohne die ein Begriff von Pädagogik nicht auskommen kann, ist die von pädagogischer Praxis und Pädagogik als deren Reflexionsinstanz bzw. Selbstbeschreibung:

Abb. 1.: Pädagogik 1 als Einheit der Unterscheidung von Praxis und Selbstbeschreibung.

\section{Pädagogik 1}

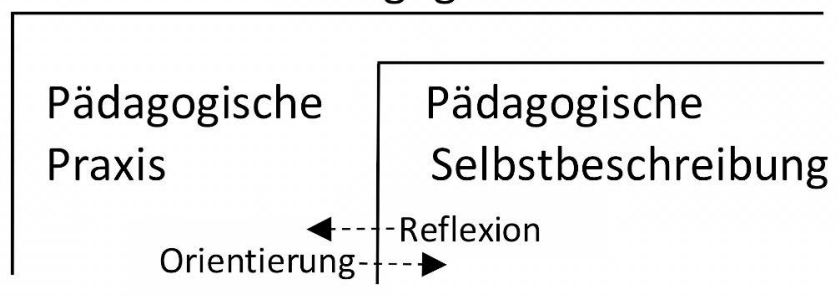

Mit >Pädagogik $1<$ ist hier ein Begriff von Pädagogik gemeint, der lediglich die Einheit der Unterscheidung von Praxis und Selbstbeschreibung formuliert und die Relation der so verstandenen Pädagogik zur Erziehungswissenschaft außen vor lässt.

Das Hilfreiche dieser Luhmannianischen Unterscheidung besteht darin, die pädagogische Selbstbeschreibung als Reflexionsinstanz der Praxis rein funktional zu fassen. Mit pädagogischer Selbstbeschreibung sind die affirmativen Semantiken gemeint, mit denen sich das konkrete pädagogische Handeln und Kommunizieren auf eine motivierende, legitimierende und orientierende Weise beschreiben kann. Diese af- 
firmativen Selbstbeschreibungen werden als funktional unverzichtbar erkannt (vgl. Luhmann 2002: 169f.). Dass diese Semantiken aus einer erziehungswissenschaftlichen Perspektive als Kitsch betrachtet werden können (vgl. Reichenbach 2003) - >Liebe für die Welt‘, >Hoffnung und >das Gute in der Welt‘, wie sie im Manifest vorkommen, scheinen hierfür gute Beispiele -, ist auf dieser Ebene völlig unerheblich. Schließlich geht es nicht darum, was derartige Semantiken für die Erziehungswissenschaft, sondern, was sie für die pädagogische Praxis leisten. Es gibt eine Reihe von Gründen, weshalb Praxis in pädagogischen Handlungsfeldern darauf angewiesen ist, sich mit »hohen Ideen« (Luhmann 2002: 169) wie Bildung, Ganzheitlichkeit, Partizipation, Inklusion, Augenhöhe, Selbstbestimmung (vgl. Krönig 2018a) etc. zu inspirieren. Die geringeren Gründe sind die Motivation, die dies freisetzt, die Legitimation z.B. von Zwang sowie die normative Handlungsorientierung in überkomplexen Situationen. Wesentlicher ist, dass pädagogische Praxis eine spezifische Form der Beobachtung und Symbolisierung benötigt, um überhaupt ihre spezifische Operativität anlaufen und fortlaufen lassen zu können. Unverzichtbar ist es beispielsweise, Kinder als bildsam anzusehen (Herbart 1984 [1835]: 5) und sich selbst eine "gute Absicht" (Luhmann 2002: 56) zuzuschreiben. Tue ich ersteres nicht, konstruiere ich, moderner gesagt, mein Gegenüber nicht als Medium der Erziehung (vgl. Luhmann 1991), sehe das Kind also nicht im Lichte seiner eigenen zugleich offenen und beeinflussbaren Möglichkeiten und habe daher keinen Grund, geduldig daraufhin zu arbeiten, dass es diese Möglichkeiten entdeckt, für sich bestimmt und ergreift. Ich könnte dann noch auf dieses Kind saufpassen bewahren, in es als Humankapital >investieren oder es wie einen Erwachsenen mit sich selbst identifizieren, ihm dadurch die volle Verantwortung für das eigenen Handeln zuschreiben und entsprechend moralische (letztlich auch juristische) Urteile fällen etc. Alle Semantiken, die damit zu tun haben, das Kind als offenen und zugleich beeinflussbaren Möglichkeitsraum zu sehen (Selbstbestimmung, Freiheit, Chancen, Potentiale, das Kind als Forscher und Entdecker usw.) und die damit verbundene >Hoffnung , formieren sozusagen die originäre pädagogische Beobachtungsweise - die Manifestautor*innen würden wohl von einem 
pädagogischen In-der-Welt-Sein sprechen, die bzw. das ohne diese semantischen Stützungen kaum aufrechtzuerhalten wäre. Jederzeit kann das Alltägliche, das Ökonomische, das Moralische, das Politische, das Therapeutische etc. in pädagogische Interaktion eindringen und das Pädagogische korrumpieren oder beenden. Erst der Verweis auf >das Kind ‘, >die Bildung « und die sich darum gruppierenden Semantiken lassen der Heteronomisierung etwas spezifisch Pädagogisches entgegensetzen. Noch deutlicher als bei der konstitutiven These der Bildsamkeit des Kindes ist es bei der Selbstaffirmation des pädagogischen Handelns. Wer in pädagogischen Interaktionssystemen keinen wie auch immer indirekten (vgl. Liegle 2009) Einfluss auf Kinder nehmen will, ist nicht pädagogisch tätig. Wer den eigenen Einfluss nicht von Indoktrination, Manipulation und Gewalt unterscheiden kann und entsprechende wie auch immer paradoxe Semantiken dafür veranschlagt (vgl. Binder et al. 2021), ebenso.

Wenn die postkritische Pädagogik also affirmative Semantiken zur Beschreibung pädagogischer Praxis ins Spiel bringt, ist das viel nüchterner bzw. funktionaler als man zunächst meinen könnte. Die Selbstaffirmation pädagogischer Praxis durch pädagogische Semantiken ist Möglichkeitsbedingung deren bloßer Aufrechterhaltung. Postkritik bedeutet an dieser Stelle, auf einen unbestreitbaren Sachverhalt hinzuweisen, der möglicherweise von der kritischen Pädagogik nicht gesehen, jedenfalls nicht betont wird. Die normative Aufladung bzw. der Kitsch, der in diesen Semantiken auftaucht, ist also auch in der postkritischen Pädagogik als pädagogische Selbstbeschreibung zu verstehen und zu verorten und ist zunächst noch nicht als Einführung dieser Semantiken in erziehungswissenschaftliche Theorie zu sehen.

Trotz seiner programmatischen Ausrichtung - vorherrschend z.B. im Schlussabschnitt - macht sich das Manifest eindeutig als wissenschaftlicher Text kenntlich. Philosophische und wissenschaftstheoretische Begriffe wie Poststrukturalismus, Postmoderne, Relativismus, Universalismus, Affirmativität vs. Kritik und Hermeneutik werden als Fachbegriffe ebenso vorausgesetzt wie eine Reihe von Autor*innen, deren Kenntnis sicherlich nicht als Professionswissen gelten kann. Es muss also geklärt werden, wie sich die postkritische Päd- 
agogik als Erziehungswissenschaft positioniert und ihr Verhältnis zu den affirmativen pädagogischen Selbstbeschreibungen (Pädagogik 1) organisiert.

Abb. 2.: Pädagogik 2 als Einheit der Unterscheidung von Pädagogik und Erziehungswissenschaft.

\section{Pädagogik 2}

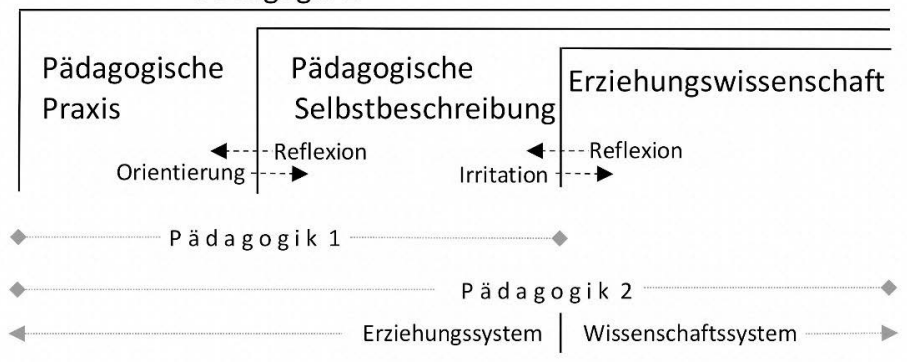

Pädagogische Selbstbeschreibungen werden nicht im Wissenschaftssystem angefertigt und sind für dieses auch nicht als wissenschaftliche Texte anschlussfähig. Gleichwohl ist eine zentrale Aufgabe der Erziehungswissenschaft, die Semantiken der Pädagogik zu beobachten, $\mathrm{zu}$ analysieren und zu kritisieren. Die Erziehungswissenschaft ist die wissenschaftliche Reflexionsinstanz der Pädagogik (1). Systemtheoretisch sieht man hier eine Systemgrenze dergestalt, dass sich die pädagogische Selbstbeschreibung zwar von wissenschaftlichen Diskursen irritieren lässt, tatsächlich aber in keiner Weise deren Operationsweise nachvollziehen kann (vgl. Krönig 2018b). Pädagogische Selbstbeschreibungen wie Konzeptionen, Ratgeberliteratur, Bildungspläne und praxisnahe Zeitschriftenartikel nehmen - insbesondere neu aufkommende - Schlüsselbegriffe aus der Erziehungswissenschaft auf, kappen dabei aber deren diskursive Zusammenhänge. Partizipation, Inklusion oder Resilienz werden dann von wissenschaftlich multiper- 
spektivisch diskutierten Problemen zu rein affirmativen Semantiken, $\mathrm{zu}$ Dingen, die >wichtigく und sgut sind und daher >umgesetzt gefördert werden müssen (vgl. Krönig 2018a). Man kann also die Einführung erziehungswissenschaftlicher Begriffe in die pädagogische Selbstbeschreibung als Positivierung bzw. Verdinglichung verstehen, wenn damit gemeint ist, dass von Differenz auf Identität umgestellt wird (vgl. Clam 2002).

Die Erziehungswissenschaft beobachtet dies und allein dieses Beobachtet-Werden durch Wissenschaft hält eine starke strukturelle Kopplung von Erziehungssystem und Wissenschaft aufrecht, jedenfalls wenn man sieht, dass besondere Irritationsbereitschaften auf beiden Seiten ausgebildet und deren Effekte bearbeitet werden (vgl. Luhmann 1998: 533). Die Frage ist nun, wie sich die postkritische Pädagogik - so wie sie im Manifest skizziert wird - als wissenschaftliche Reflexionsinstanz der Pädagogik aufstellt. Wie ist ihr affirmativer Standpunkt zu den affirmativen Semantiken in pädagogischen Selbstbeschreibungen $\mathrm{zu}$ verstehen? Wie oben schon angedeutet, kann die Einsicht in die funktionale Notwendigkeit affirmativer Semantiken auf der Selbstbeschreibungsebene der Pädagogik ganz nüchtern und distanziert analysiert werden. Man kann also fragen, was mit der pädagogischen Praxis geschieht, wenn sie sich nicht selbst affirmiert und keine Selbstbeschreibungen vorliegen, die dies >professionalisieren . Die Antwort, die die Autor*innen des Manifests geben, liegen im Wesentlichen im Bereich der Heteronomisierung, d.h. Autonomiegefährdung pädagogischer Praxis. Systemtheoretisch lässt sich diese Antwort nachvollziehen, ohne überhaupt Stellung zu den bedrohten oder bedrohenden Werten nehmen zu müssen: Wenn sich überhaupt Erziehung als Funktionssystem ausbildet und stabilisiert, dann nur, insofern eine Reihe von Strukturen aufrecht erhalten werden, die ihre Differenz zur Umwelt reproduzieren bzw. reproduzierbar machen. Eine dieser Strukturen von nicht $\mathrm{zu}$ unterschätzender Bedeutung (vgl. Stäheli 1998) ist, wie gezeigt, die affirmative Selbstbeschreibung. Irritierend ist aber, jedenfalls systemtheoretisch, dass die Autor*innen des Manifests eine Wir-Position formulieren, die sich nicht allein auf die Autor*innenschaft bezieht, sondern vielmehr die Einheit von 
pädagogischer Praxis und erziehungswissenschaftlicher Reflexion $\mathrm{zu}$ behaupten scheint:

Um die Rolle der Pädagog*in etwas positiver darzustellen, als eine Initiation der neuen Generationen in eine gemeinsame Welt, eröffnen wir die Idee einer post-kritischen Pädagogik, die die Liebe zur Welt einfordert. Dies ist keine Akzeptanz der Dinge, wie sie sind, sondern eine Bejahung der Wertigkeit dessen, was wir in der Cegenwart tun und der Dinge, von denen wir annehmen, dass sie es wert sind, weitergegeben zu werden. (Hodgson et al. 2022: 23)

Die, die in der Praxis eine neue Generation in eine gemeinsame Welt einführen, sind offenbar die gleichen, die eine Idee von postkritischer Pädagogik formulieren. Ist das pädagogisch->liebende< In-der-WeltSein also das Gleiche wie dessen wissenschaftliche Analyse? Das ständig auftretende >Wir verführt zu dieser Deutung, zu der man sich allerdings nicht leichterdings hinreißen lassen sollte. Hermeneutisch ist schließlich das geboten, was man heute »steelmanning " (Messinger 2012, o.S.) in Abgrenzung zum »strawmanning (ebd.) nennt: »the art of addressing the best form of the other person's argument, even if it's not the one they presented« (ebd.). Die zugegeben naheliegende Deutung der Einheit von pädagogischem und wissenschaftlichem Inder-Welt-Sein ist wohl in diesem Geiste auszuschließen. Das Manifest ist schließlich wesentlich ein Apell, die Autonomie des Pädagogischen $\mathrm{zu}$ erkennen, $\mathrm{zu}$ bejahen und wiederzugewinnen. Das ist zwar in erster Linie gegen eine Politisierung gerichtet, aber im Grunde geht es darum, »Bildung um der Bildung willen zu verteidigen« (Hodgson et al. 2022: 24) gegen jegliche Art der Verzweckung und Instrumentalisierung (ebd.). Diese Position der Immanenz verbietet nicht nur eine Politisierung und eine Ökonomisierung, sondern konsequenterweise auch eine Verwissenschaftlichung des Pädagogischen. Letztlich lässt sich diese Position zudem nur vertreten, wenn auf der anderen Seite auch die Autonomie der Wissenschaft (und der Politik usw.) ebenso affirmiert wird. Hier wird deutlich, dass das Manifest zugleich den Kern einer Gesellschaftstheorie enthält, der systemtheoretisch entwickelt werden könnte. Die bekannte »Nüchternheit und Präzision« (Marius/Jahraus 
1997: 70), mit der Niklas Luhmann die funktionale Differenzierung der gesellschaftlichen Systeme vorträgt, scheint weder post-kritisch noch in irgendeiner Weise affirmativ zu sein. Gleichwohl sind Autopoiesis, die Orientierung an der eigenen Funktion, die spezifische binär codierte Beobachtungsweise und die operative Geschlossenheit Theoreme, die die harte Differenzierung zwischen Pädagogik ${ }^{1}$ und den anderen Funktionssystemen herstellen und dadurch die Autonomie-These des Manifests teilen und begründen können. Auch für die Systemtheorie wäre eine Politisierung der Pädagogik als Korruption anzusehen und eine Entdifferenzierung (z.B. in der Form sdas Pädagogische ist per se politisch $\varsigma$ ) als Krise, die die Funktion und die Leistungen des Erziehungssystems massiv gefährdeten (vgl. Radtke 2019: 340). Orthodoxe Systemtheoretiker*innen würden wohl die Frage "Soll man sich für den Erhalt der Differenzen zwischen den Systemen, ihrer Eigenlogiken und konkurrierende Eigennormativitäten einsetzen - schon weil das Prinzip der Differenzierung strukturell erhalten und der Kollaps auch nur einzelner Systeme vermieden werden soll?« (Radtke 2019: 340f.) als ungültig von der Hand weisen. Es handelt sich schließlich um eine moralische Frage, die für wissenschaftliche Beobachter nicht anschlussfähig ist. Darüber hinaus ist die Idee, Personen könnten sich gesellschaftsstrukturell >einsetzen`, nicht nachvollziehbar.

Hinsichtlich der Frage, wie sich die Normativität der postkritischen Pädagogik, insofern man sie als eine erziehungswissenschaftliche versteht, zu der pädagogischen Normativität (»Liebe für die Welt«) verhält, können wir nun sehen, dass es sich um ganz verschiedene Normativitäten handelt:

Keineswegs muss man also annehmen, die postkritische Erziehungswissenschaft verhalte sich >liebevolk zu ihren Gegenständen. Sie kann vielmehr einen abstrakten und minimalen Standpunkt vertreten: Wenn es überhaupt eine Pädagogik im Sinne einer spezifischen Beobachtungs-, Kommunikations- und Interaktionsweise, einer eigenen Profession, oder, wie es die postkritische Pädagogik sagen würde:

1 Die Systemtheorie spricht vom Erziehungssystem und meint mit Pädagogik die Selbstbeschreibung der Erziehungspraxis (s. Abb. 1). 
Abb. 3.: Drei Affirmativitätsformen der postkritischen Pädagogik.

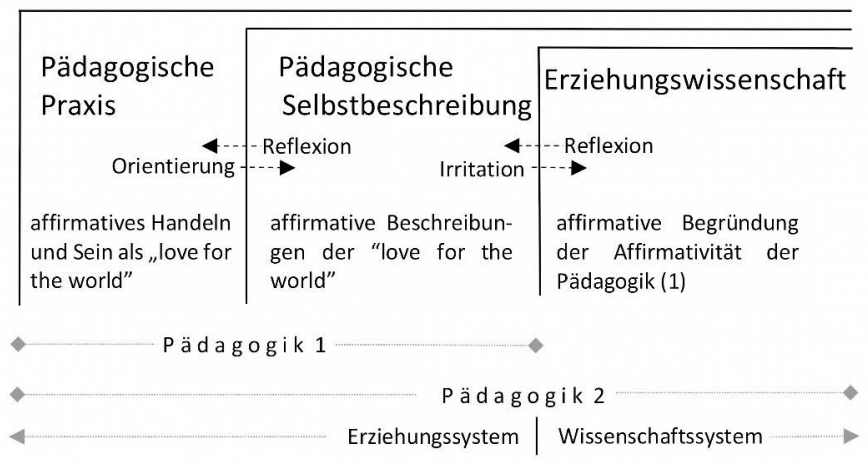

eines originären In-der-Welt-Seins gibt, dann nur, insofern und solange sie sich von anderen Modi abheben kann bzw. gegen deren Einflüsse abgrenzt. Ob es sie geben sollte, ob wir also in einer modernen, funktional differenzierten Gesellschaft mit spezialisierten Institutionen leben wollen oder nicht, ist eine politische Frage, zu der sich seit der Aufklärung wohl sämtliche, aber gerade aktuelle erziehungswissenschaftliche Ansätze positionieren (vgl. Oelkers 2015). Dass hier die postkritische Pädagogik mit einem klaren Ja antwortet, macht sie als Erziehungswissenschaft nicht auffällig normativ oder affirmativ. Das Argument nämlich, das sie dazu führt, ist keineswegs politisch oder auch nur normativ. Dass die Affirmation der eigenen professionellen Praxis nur im Rückgriff auf affirmative Semantiken im Gefüge einer relativ kohärenten Selbstbeschreibung gelingt, kann systemtheoretisch bzw. strukturfunktionalistisch herausgearbeitet werden (vgl. Krönig 2022). Ob pädagogische Praxis ohne Selbstaffirmation auskommen kann, ist zumindest zweifelhaft. Wie eingangs skizziert, kann von Pädagogik, die ihre eigenen Einflussnahmeversuche nicht selbstreferentiell mittels affirmativer Semantiken von Manipulation, Dressur und Indoktrination unterscheidet, nicht sinnvoll gesprochen werden. Die erziehungswissenschaftliche Begründung der Affirmativität der 
Pädagogik ist also zwar affirmativ, kommt aber mit einer bloßen nahezu formalen - Affirmation der funktional differenzierten Gesellschaft aus, um daraus die Autonomie der Pädagogik abzuleiten, aus der wiederum die funktionale Notwendigkeit affirmativer Semantiken abgeleitet werden kann. Das theoretische Pendant zur Spezifik des Erziehungssystems als ein System in der funktional differenzierten Gesellschaft ist in der postkritischen Pädagogik das Pädagogische in Abgrenzung zum Politischen, Ökonomischen, Religiösen, Wissenschaftlichen etc. Diese Bereiche werden nicht soziologisch, sondern philosophisch über je spezifische Formen des In-der-Welt-Seins differenziert. Dieser Sachverhalt bezieht sich also auf die Ebene der Bezugswissenschaft, auf die die Erziehungswissenschaft als Irritationsquelle zurückgreift, d.h. um sich mit Konzepten zu stimulieren, deren Gesamtzusammenhang in anderen Disziplinen komplexitätsadäquat diskutiert wird und die daher in der Erziehungswissenschaft zugleich anschließen und sich entziehen, mithin Reibung erzeugen. $\mathrm{Da}$ sich die Erziehungswissenschaften gezielt auf Irritationen aus verschiedenen Bezugswissenschaften einstellt, bleibt sie sozusagen im Diskurs, partizipiert an verschiedenen Wissenserzeugungsprozessen und sieht sich dadurch in gewisser Weise wissenschaftlich (z.B. soziologisch, psychologisch, philosophisch) >fundiertく.

Abb. 4.: Pädagogik als Einheit der Differenz von Reflexionsverhältnissen.

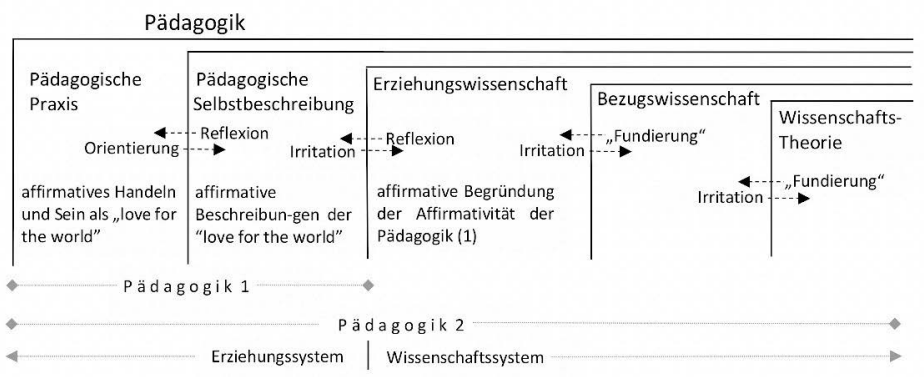


Dass die Bezugswissenschaft im Falle der postkritischen Pädagogik im Wesentlichen die Philosophie ist und dabei im Besonderen ontologische Ansätze Berücksichtigung finden, kann die aktuelle eher sozialwissenschaftlich geprägte Erziehungswissenschaft irritieren. Ob der philosophische, in diesem Falle auch ontologische Fundierungsversuch haltbar ist, müsste auf der wissenschaftstheoretischen Reflexionsebene diskutiert werden. Die Kontingenz selektiven Zugriffs auf bezugswissenschaftliche Diskurse, die prinzipiell nie komplexitätsadäquat >importiert ‘ werden können, ist ein grundsätzliches Problem der Erziehungswissenschaft. Schlichte Verweise darauf, dass sich die postkritische Pädagogik ontologisch $\mathrm{zu}$ fundieren sucht und dass dies einer Essentialisierung gleichkomme (vgl. Hodgson 2020), erreichen jedenfalls selber nicht die wissenschaftstheoretische Reflexionsebene.

Aus all dem folgt, dass jede Kritik der postkritischen Pädagogik, die deren Affirmativität auf der Grundlage deren pädagogischer Semantiken in den Blick nimmt, ihr Ziel verfehlt. Genauso könnte man jeden Entwurf einer Pädagogik nach dem beurteilen, was praxisnah z.B. in Ratgebern oder Konzeptionen über diese geschrieben wird. Auch pädagogische Ansätze, die sich kritisch nennen, sind - wie gezeigt - sozusagen auf motivierende, orientierende, um nicht zu sagen: erbauliche und kitschige Semantiken angewiesen, die in diesem Fall aus dem aktivistischen Genre stammen und beispielsweise mit apokalyptischen Bildern die Leser*innen zum Erschauern bringen (vgl. Peters 2020) oder Formeln wie Gerechtigkeit (vgl. Papastephanou et al. 2020) an die Stelle von nur scheinbar affirmativeren Semantiken wie Freiheit oder Bildung setzen. Auch dies ist auf der Ebene der pädagogischen Selbstbeschreibungen nicht zu kritisieren. Die Kritik muss sich hier wie da in die andere Richtung wenden und die Reflexionsinstanzen der Bezugswissenschaften und der Wissenschaftstheorie aufrufen. Die Auseinandersetzung mit der Philosophie und wissenschaftstheoretischen Grundlage der postkritischen Pädagogik steht noch aus und kann nicht durch Geschmacksfragen (welche Semantiken auf der Selbstbeschreibungsebene mehr oder weniger zusagen) ersetzt werden. 


\section{Literatur}

Binder, Ulrich; Heidenwag, Jari (2013): »»Also sprach Janusz Korczak...« Eine Analyse von Korczaks Argumentationsarchitektur«, in: Rosemarie Godel-Gaßner, Sabine Krehl: (Hg.): Facettenreich im Fokus. Janusz Korczak und seine Pädagogik - historische und aktuelle Perspektiven. Jena: Garamond-Verlag: S. 243-356.

Binder, Ulrich; Krönig, Franz Kasper; Tenorth, Heinz-Elmar (2021): »Die Produktivität des Paradoxen. Eine Heuristik für erziehungswissenschaftliche »Euryalistik«, in: Ulrich Binder, Franz Kasper Krönig (Hg.): Paradoxien (in) der Pädagogik. Weinheim: Beltz Juventa: S. 14-51.

Clam, Jean (2002): Was heißt, sich an Differenz statt an Identität orientieren? Zur De-ontologisierung in Philosophie und Sozialwissenschaft. Konstanz: UVK.

Herbart, Johann Friedrich (1984 [1835]): Johann Friedrich Herbart. Band 1. Umriß pädagogischer Vorlesungen, besorgt von Josef Esterhues. Paderborn: Schöningh.

Hodgson, Naomi (2020): »Post-critique, politics, and the political in educational philosophy«, in: On Education. Journal for Research and Debate, 3 .

Hodgson, Naomi; Vlieghe, Joris, Zamojski, Piotr (2017): Manifesto for a Post-Critical Pedagogy. Earth (Milky Way): punctum books.

Hodgson, Naomi; Vlieghe, Joris; Zamojski, Piotr (2022): »Manifest für eine Post-Kritische Pädagogik«, in: Martin Bittner, Anke Wischmann (Hg.): Kritik und Post-Kritik. Zur deutschsprachigen Rezeption des »Manifests für eine Post-Kritische Pädagogik«. Bielefeld: transcipt Verlag: S. 19-24.

Krönig, Franz Kasper (Hg.) (2018a): Kritisches Glossar Kindheitspädagogik. Weinheim: Beltz Juventa.

Krönig, Franz Kasper (2018b): »Modernisierungserzählungen in der Pädagogik. Ein systemtheoretischer Orientierungsversuch mit ideologiekritischer Wendung«, in: Ulrich Binder (Hg.): Modernisierung und Pädagogik - ambivalente und paradoxe Interdependenzen. Weinheim: Beltz Juventa: S. 31-44. 
Krönig, Franz Kasper (2020): »Auswege aus dem Macht-Äther. Post-kritische Friedenspädagogik als Beispiel autonomer Kindheitspädagogik «, in neue praxis (5) 20, 2020: S. 481-491.

Krönig, Franz Kasper (2022): »Ist die Kindheitspädagogik ökonomisierbar? Zur Frage der Autonomie kindheitspädagogischer Kommunikation als Bedingung von Ökonomisierung«, in: Johanna Mierendorff, Thomas Grunau, Thomas Höhne (Hg.): Der Elementarbereich im Wandel. Weinheim: Beltz Juventa: S. 90-105.

Liegle, Ludwig (2009): »Wir brauchen eine Didaktik der indirekten Erziehung«, in: Betrifft Kinder (9) 9: S. 6-13.

Luhmann, Niklas (1991): »Das Kind als Medium der Erziehung«, in Zeitschrift für Pädagogik, 37 (91) 1: S. 19-40.

Luhmann, Niklas (1998): Die Gesellschaft der Gesellschaft. Frankfurt a.M.: Suhrkamp.

Luhmann, Niklas (2002): Das Erziehungssystem der Gesellschaft. Frankfurt a.M.: Suhrkamp.

Marius, Benjamin/Jahraus, Oliver (1997): Systemtheorie und Dekonstruktion. Die Supertheorien Niklas Luhmanns und Jacques Derridas im Vergleich. Siegen: Lumis.

Messinger, Chana (2012): Knocking Down a Steel Man: How to Argue Better The Merely Real [Online]. Available from: https://themer elyreal.wordpress.com/2012/12/07/steelmanning/[Abfragedatum: 07.12.2012 2021].

Oelkers, Jürgen (2015): »Ist die Erziehungswissenschaft politisch?«, in: Erziehungswissenschaft, 26 (50): S. 37-44.

Papastephanou, Marianna; Zembylas, Michalinos; Bostad, Inga; Oral, Sevget Benhur; Drousioti, Kalli; Kouppanou, Anna; Strand, Torill; Wain, Kenneth/Peters, Michael A./Tesar, Marek (2020): »Philosophy of education in a new key: Education for justice now «, in Educational Philosophy and Theory, August 2020: S. 1-16.

Peters, Michael A. (2020): »The Armageddon Club: education for the future of humanity«, in: Educational Philosophy and Theory, 52: S. 816-819.

Radtke, Frank-Olaf (2019): »Konkurrierende Eigennormativitäten«, in: Wolfgang Meseth, Rita Casale, Anja Tervooren, Jörg Zirfas (Hg.): 
Normativität in der Erziehungswissenschaft. Wiesbaden: Springer VS: S. 329-342.

Reichenbach, Roland (2003): »Pädagogischer Kitsch«, in Zeitschrift für Pädagogik, 49 (6): S. 775-789.

Stäheli, Urs (1998): »Die Nachträglichkeit der Semantik. Zum Verhältnis von Sozialstruktur und Semantik«, in: Soziale Systeme, 4: S. 315-339. Vlieghe, Joris; Zamojski, Piotr (2020): »Towards an immanent ontology of teaching Leonard Bernstein as a case-study«, in: Ethics and Education, 15: S. 1-17. 
